\title{
IN VIVO BIOSYNTHESIS AND TRANSPORT OF OXYTOCIN, VASOPRESSIN, AND NEUROPHYSINS TO POSTERIOR PITUITARY AND NUCLEUS OF THE SOLITARY TRACT ${ }^{1}$
}

\author{
JEFFREY D. WHITE, ${ }^{2}$ JAMES E. KRAUSE, and JEFFREY F. McKELVY \\ Department of Neurobiology and Behavior, State University of New York, Stony Brook, New York 11794
}

Received August 5, 1983; Revised November 11, 1983; Accepted November 15, 1983

\begin{abstract}
A growing body of literature suggests that oxytocin (OXY) and arginine-vasopressin (AVP), in addition to their neuroendocrine roles, may serve as neuromodulators within the central nervous system of mammals. The present study investigated the biosynthesis of OXY, AVP, and their associated neurophysins in the paraventricular nucleus of the hypothalamus (PVN) and the transport of these peptides to the neural lobe and the nucleus of the solitary tract (NTS) in the brainstem of the rat.

Rats were cannulated bilaterally in the PVN, and $24 \mathrm{hr}$ later a 2-hr pulse of ${ }^{35}$ S]cysteine was administered using an Alzet minipump delivery system. After a 10-hr chase period, the neural lobe was removed and the PVN and NTS were punched. Tissue homogenates were adsorbed to and eluted from octadecyl-silica cartridges and analyzed by linear high performance liquid chromatography (HPLC) gradient elution, chemical and enzymatic modification, and exponential gradient HPLC elution followed by linear HPLC gradient analysis using an ion-pairing buffer system.

This rigorous approach has allowed us to identify ${ }^{35} \mathrm{~S}$-labeled material which co-purifies with OXY and AVP from the PVN, neural lobe, and NTS samples. Specific transport of the nonapeptides to the NTS was demonstrated when a unilateral transection of the hypothalamic fibers resulted in a unilateral depletion of the radiolabeled peptides from the NTS samples. Additionally, each of the neurophysins was purified from the neural lobe and NTS samples after linear HPLC gradient analysis, ion-pairing buffer linear gradient analysis, then tryptic digestion followed by exponential gradient HPLC analysis. These data demonstrate that studies on the physiological regulation of AVP and OXY biosynthesis for both endocrine and autonomic projections are now possible in individual animals.
\end{abstract}

The hypothalamoneurohypophysial system has been studied extensively as a classical neuroendocrine unit. Neurons in the supraoptic (SON) and paraventricular nuclei (PVN) synthesize the nonapeptide hormones arginine-vasopressin (AVP) and oxytocin (OXY) and their associated neurophysins. These peptides are transported to terminal fields in the neural lobe of the hypophysis (posterior pituitary), from which they are released into the general circulation for delivery to distant target tissues such as the kidney, uterus, and mammary gland (Scharrer and Scharrer, 1954; Sachs, 1969). Recently,

${ }^{1}$ This work was supported by National Science Foundation Grant BNS 792181 (J. F. M.) and National Institutes of Health Grant NS 18942 (J. E. K.). We gratefully acknowledge the following people for their assistance: Dr. Miklos Palkovits for performing the lesions of the hypothalamic fibers, Dr. J. P. Advis for helpful discussions regarding the cannulations, and J. Demian for the illustrations.

${ }^{2}$ To whom correspondence should be addressed. however, neuroanatomical studies utilizing retrograde tracer and immunohistochemical techniques have demonstrated that immunoreactivity corresponding to OXY, AVP, and neurophysins can be detected in neurons projecting from the PVN to the neuropil of the medulla, especially the nucleus of the tractus solitarius (NTS), and the spinal cord, including the autonomic preganglionic cell column (Swanson and Kuypers, 1980; Sawchencko and Swanson, 1982a). The implications of these findings are of great importance; i.e., they suggest that integration of both endocrine and autonomic responses may occur within a single nucleus and involve the elaboration of the same chemical signal to both neurohemal and synaptic sites. However, before detailed physiological and electrophysiological studies can be initiated to pursue the implications of these neuroanatomical findings, biochemical characterization of the molecular species, derived from the processing of the precursors to vasopressin and oxytocin, which are transported to syn- 
aptic sites in these descending projections, must be carried out.

In this report, we present biochemical evidence related to this point. We have utilized in vivo methods for studying neuropeptide biosynthesis and transport which we have found to be useful in the study of other neuropeptides (McKelvy et al., 1983). Thus, after administration of radiolabeled amino acid into the PVN, radiolabeled peptides were harvested from the posterior pituitary and the NTS which co-purify with synthetic AVP and $\mathrm{OXY}$, and rat neurophysins, through sequential purification steps.

\section{Materials and Methods}

Materials. Sodium phosphate, phosphoric acid, high performance liquid chromatography (HPLC)-grade acetonitrile, formic acid $(88 \%)$, hydrogen peroxide $(30 \%)$, and Scinti-Verse II were from Fisher Scientific Co. Triethylamine, trifluoroacetic acid (TFA), dithiothreitol, and L-1 -tosylamide-2-phenylethyl chloromethyl ketone (TPCK)-treated trypsin were from Sigma Chemical Co. Reverse phase (C8) HPLC columns were from Brownlee. The HPLC system was Hewlett-Packard model 1084B. HPLC-grade water was obtained from a Milli-Q water system from Millipore Corp. $\left[{ }^{35} \mathrm{~S}\right]$ Cysteine (specific activity $>1000 \mathrm{Ci} / \mathrm{mmol}$ ) was from New England Nuclear. Rat posterior pituitaries were from A. F. Parlow through the National Institutes of Health Pituitary Program.

Cannulation and label administration. Male SpragueDawley rats (175 to $225 \mathrm{gm}$ ) were cannulated bilaterally over the PVN (A $0.2 \mathrm{~mm}, \mathrm{~L} 0.5 \mathrm{~mm}$, and V $7.3 \mathrm{~mm}$, with respect to bregma) under ether anesthesia. After a recovery period of at least $18 \mathrm{hr}$, the rats were again ether anesthetized, and $250 \mu \mathrm{Ci}$ of $\left[{ }^{35}\right.$ S $]$ cysteine in $3 \mu \mathrm{l}$ of artificial brain extracellular fluid (ECF) (Elliot, 1969) were loaded into each cannula. The cannulae were connected via polyethylene tubing filled with ECF to osmotic minipumps (Alza Corp.) which pumped at a flow rate of $1 \mu \mathrm{l} / \mathrm{hr}$. One microliter of the amino acid solution was preloaded into the infusion site, and the remaining $2 \mu \mathrm{l}$ were pumped in over the next $2 \mathrm{hr}$. After a 10-hr chase period, the animals were sacrificed by decapitation, and the brains were rapidly removed and frozen. The neural lobe was dissected from the unfrozen hypophysis. The PVN and NTS were punched from frozen coronal brain sections. All tissues were homogenized in ice-cold $2 \mathrm{M}$ acetic acid twice over a $24-\mathrm{hr}$ period, to give a total volume of $1 \mathrm{ml}$. Twenty-five micrograms each of carrier AVP, OXY, and somatostatin-14 (SS-14) were added at the time of homogenization. SS-14 was added as an additional internal standard because it eluted from the first HPLC system near the elution positions of the neurophysins. To promote reproducibility, all infusions began at 12:00 P.M.

Peptide purification. The supernatants from the acid extracts were applied to $\mathrm{C}_{18}$ SEP-PAK cartridges (Waters Associates) and then were eluted with $3 \mathrm{ml}$ of propanol/0.1\% TFA (1:1) into 15-ml conical bottom polypropylene tubes, followed by lyophilization. The peptides were resuspended in $100 \mu \mathrm{l}$ of $0.1 \%$ TFA and applied to the first HPLC system (system 1): Buffer A $(0.1 \mathrm{M}$ monobasic sodium phosphate, $0.1 \mathrm{M}$ phosphoric acid, $\mathrm{pH}$ 2.3)/acetonitrile; linear gradient from 5 to $45 \%$ acetonitrile over $60 \mathrm{~min}$ at a flow rate of $1.0 \mathrm{ml} / \mathrm{min}$. Oneminute fractions were collected. The elution position of the carrier peptides was monitored by absorption at 210 $\mathrm{nm}$. Carrier recovery estimations were based on digital integration of peak areas and comparisons to known quantities of standards. Each peak could be collected in a total volume of $2 \mathrm{ml}$. Aliquots of 50 to $100 \mu \mathrm{l}$ were routinely taken for scintillation counting. The collected nonapeptide carrier-containing fractions were directly lyophilized and then resuspended in $50 \mu \mathrm{l}$ of $0.1 \%$ TFA. AVP and OXY were oxidized separately, using $50 \mu \mathrm{l}$ of freshly prepared performic acid (Hirs, 1967) on ice for $90 \mathrm{~min}$. At the end of the incubation the peptides were injected directly onto the second HPLC system. For each of the peptides an exponential gradient was used (see McKelvy et al., 1983), and in each case the elution was carried out for $5 \mathrm{~min}$ at initial conditions. For AVP, the gradient was 5 to $18 \%$ acetonitrile and for OXY the gradient was 5 to $22 \%$ acetonitrile; both gradients were run for $40 \mathrm{~min}$ at $1.0 \mathrm{ml} / \mathrm{min}$. The fractions corresponding to the elution time of the performic acid oxidized carrier were collected and lyophilized. The third HPLC system used an ion-pairing buffer, triethylammonium phosphate (TEAP: $5.2 \mathrm{ml}$ of triethylamine mixed with $5.7 \mathrm{ml}$ of phosphoric acid and brought to $500 \mathrm{ml}$ with water), and acetonitrile in a linear gradient. For AVPsulfoxide the gradient was from 5 to $40 \%$ acetonitrile and for OXY-sulfoxide the gradient was from $5 \%$ to $30 \%$ acetonitrile. Both gradients were for $40 \mathrm{~min}$ at a flow rate of $1.0 \mathrm{ml} / \mathrm{min}$ and used $20 \mathrm{ml}$ of TEAP/liter of water. From 100 to $1000 \mu \mathrm{l}$ of these fractions were then taken for scintillation counting in $5 \mathrm{ml}$ of Scinti-Verse II.

Neurophysin purification. Neurophysins were purified from neural lobe and NTS samples using conditions slightly modified from those described above for the purification of the nonapetides. Carrier unlabeled crude neurophysins (i.e., acid-soluble posterior pituitary proteins retained by a lysine-vasopressin affinity column) were added to the acid extracts and somatostatin was omitted. HPLC system 1 was again a linear gradient of Buffer A/acetonitrile with a 10 -min isocratic step added at $28.5 \%$ acetonitrile to achieve more complete resolution of the neurophysins. Carrier peaks corresponding to neurophysin II + III and neurophysin I were collected separately; 5 to $10 \%$ of the sample was taken for radioactivity determination and the remainder was lyophilized. Each sample was then applied to an ion-pairing HPLC system:TEAP $\left(150 \mathrm{ml}\right.$ of stock solution, $850 \mathrm{ml}$ of $\left.\mathrm{H}_{2} \mathrm{O}\right)$ / acetonitrile for $5 \mathrm{~min}$ at $5 \%$ acetonitrile which then rose to $45 \%$ acetonitrile over $40 \mathrm{~min}$. The carrier peptide peak was collected, an aliquot was taken for radioactivity determination, and the remainder was lyophilized. These samples were resuspended in $100 \mu \mathrm{l}$ of $0.1 \mathrm{M}$ ammonium bicarbonate/1 mM EDTA buffer, and the $\mathrm{pH}$ was adjusted to 8 with ammonium hydroxide. The samples were incubated with trypsin at an enzyme:substrate ratio of 1:30 for $120 \mathrm{~min}$ at $37^{\circ} \mathrm{C}$. The reaction was terminated by freezing. Trypsin-treated samples were applied to an exponential HPLC gradient of Buffer A/acetonitrile: $10 \%$ acetonitrile for $5 \mathrm{~min}$, rising to $31 \%$ acetonitrile in 
$40 \mathrm{~min}, 5 \mathrm{~min}$ at $31 \%$ acetonitrile, then rising to $40 \%$ acetonitrile in $5 \mathrm{~min}$. Fractions corresponding to carrier peptide peaks were taken for scintillation counting.

Neurophysin immunoprecipitation. The neurophysincontaining fractions from HPLC 1 , in the absence of added carrier, from the NTS and neural lobe samples were pooled, desalted over SEP-PAK, and lyophilized. The samples were resuspended in $200 \mu \mathrm{l}$ of immunobinding buffer (IBB) $(50 \mathrm{mM}$ sodium phosphate, $0.15 \mathrm{M} \mathrm{NaCl}$, $2 \%$ Triton $\mathrm{X}-100,1 \%$ sodium deoxycholate, $\mathrm{pH} 8.0$ ), and di-isopropylfluorophosphate (1 $\mathrm{mM})$ and pepstatin (10 $\mu \mathrm{M})$ were added to inhibit proteolysis. Ten microliters of anti-neurophysin antiserum (RN-4; a gift of Dr. A. Robinson, University of Pittsburgh) were added to each tube, and the samples were incubated overnight at $4^{\circ} \mathrm{C}$. Each sample was applied to a 100- $\mu$ l Protein A-Sepharose column, washed with IBB and PBS, and eluted with 1 $\mathrm{ml}$ of $1 \mathrm{M}$ acetic acid, and carrier neurophysin was added. The acid eluates were lyophilized, resuspended in $100 \mu \mathrm{l}$ of $0.1 \%$ TFA, and applied to the exponential HPLC gradient system described above. Fractions co-migrating with carrier neurophysins were taken for radioactivity determination.

NTS lesion. Lesions of the right solitary tract were performed at the National Institutes of Health by Dr. M. Palkovits. Under ether anesthesia, the scalp behind the skull was opened and the dura covering the brainstem was opened. A single sagittal cut was made lateral to the NTS but medial to the solitary tract at the level of the NTS to sever the fibers from the hypothalamus as they sweep up into the solitary nucleus. The cut did not penetrate the fibers leading to the spinal cord. The wound was closed with wound clips and the animals were allowed to recover for 10 to 14 days.

Sham-operated animals were prepared with the exclusion of the knife cut. The animals were cannulated and the $\left[{ }^{35}\right.$ S $]$ cysteine was administered as above. At the time of tissue harvest, the right and left NTS were punched separately as were the right and left PVN. Peptides were purified as above.

\section{Results}

Sprague-Dawley rats were bilaterally cannulated over the PVN and infused with $500 \mu \mathrm{Ci}$ of $\left[{ }^{35} \mathrm{~S}\right]$ cysteine over $2 \mathrm{hr}$. Then, after an additional $10 \mathrm{hr}$, to allow for uptake of the amino acid, synthesis and processing of the peptides, and transport, the animals were sacrificed, tissues harvested and peptides extracted. Figure 1 shows the HPLC profile of the extracted peptides, eluted from the SEP-PAK, exhibited by the neural lobe and NTS. The radioactive profile of the PVN sample was as complex as the NTS profile and is not shown for the sake of brevity. The radioactive profiles from the three regions were remarkably different. Clear peaks of radioactivity that co-migrated with the carrier AVP and OXY were apparent in the neural lobe, as were the neurophysins, which were present in sufficient quantity in the neural lobe to appear as UV-visible peaks. A clear separation of radioactive peaks corresponding to the peptides of interest from the base line of radioactivity was much less evident
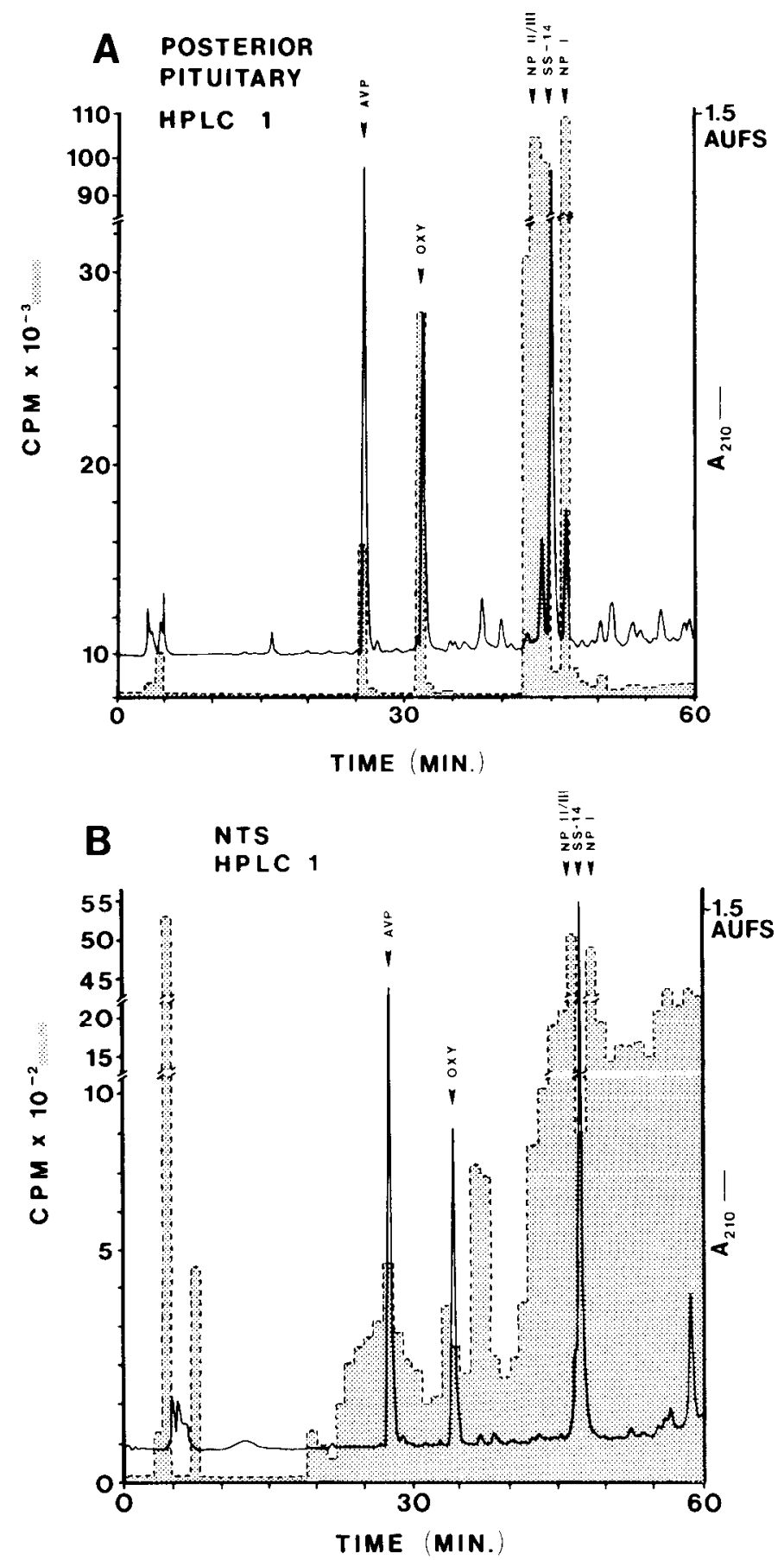

Figure 1. A representative high performance liquid chromatogram of the acid soluble proteins retained by $\mathrm{C}_{18}$ SEP-PAK cartridges. The rat was cannulated above the PVN and $500 \mu \mathrm{Ci}$ of $\left[{ }^{35} \mathrm{~S}\right]$ cysteine were administered as described under "Materials and Methods." The HPLC gradient system is system 1, described under "Materials and Methods." One hundred percent of the SEP-PAK retentate was applied for fractionation. ${ }^{35} \mathrm{~S}$ radioactivity is plotted on the left axis and represents the total amount of radioactivity present in each fraction; absorbance at $210 \mathrm{~nm}$ is plotted on the right axis $(1.5$ absorbance units full scale $=1.5$ AUFS). The elution positions of the carrier AVP, OXY, and SS-14 and endogenous neurophysins $(N p I I / I I I ; N p I)$ are marked. $A$, Posterior pituitary tissue sample; $B$, nucleus of the solitary tract (NTS) tissue sample. 
in the hypothalamic and brainstem samples. The recovery of the carrier peptides, from the initial $25 \mu \mathrm{g}$ through this first HPLC step was AVP $=79 \%(n=33), \mathrm{OXY}=$ $80 \%(n=33)$, and SS- $14=86 \%(n=23)$. No significant difference was found in the recovery of carrier from each of the three regions. Using these labeling and transport times, the ${ }^{35} \mathrm{~S}$-labeled peptides in the neural lobe appeared to be remarkably pure as suggested by the ratios of the radioactivity in the nonapeptide peaks compared to the radioactivity in the neurophysin peaks. The neurophysins each contain seven cystines, whereas the nonapeptides each contain one. With the expected molar ratio of 1 ncurophysin: 1 nonapeptide for animals of this age (Sinding et al., 1980), the observed radioactivity ratios were approximately in the expected ratio of $7: 1$.

It is apparent from the profiles in Figure 1 that further purification was needed to verify the existence of AVP and OXY in the PVN and NTS samples. The second purification step for each of the peptides incorporated both chemical modification and an exponential HPLC gradient shape to achieve better separation of the peptides from contaminating peptides. The disulfide bonds of the hormones are susceptible to performic acid oxidation which will significantly alter the charge of the peptide and, thus, its retention time on the reverse phase HPLC column, and will also cleave any mixed disulfides which may have formed during the purification. Figures 2 and 3 illustrate the radioactive profiles from the neural lobe and PVN samples for both AVP and OXY after performic acid oxidation. As the figures demonstrate, the peptides were significantly altered in their retention

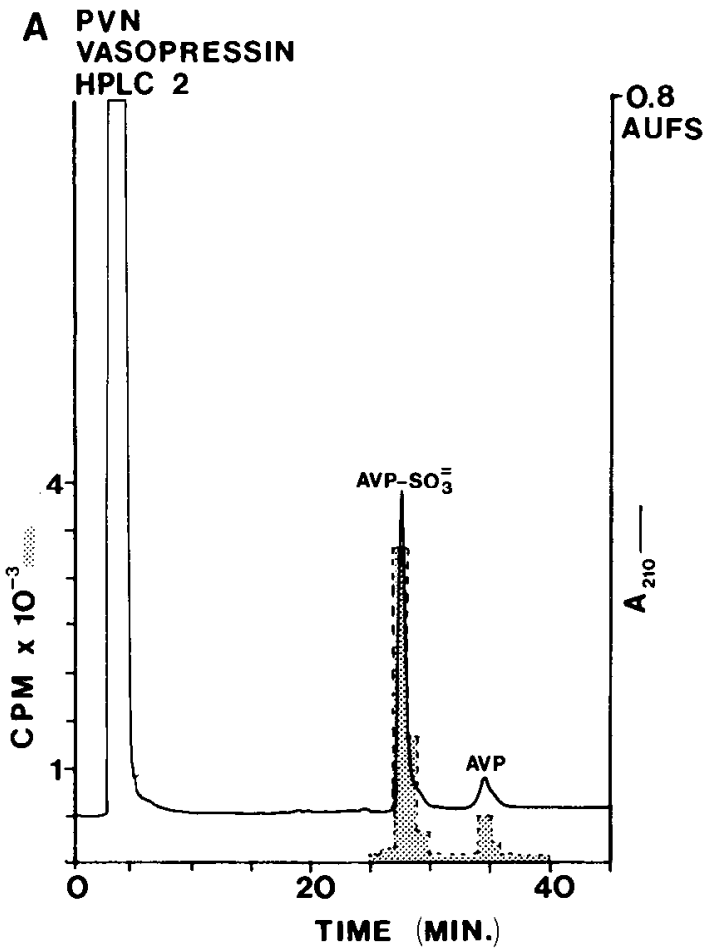

times when compared to the native peptides. The radioactive profiles for the NTS samples are not shown because an accurate radioactivity measurement could only be made on a significant aliquot of the sample. Therefore, aliquots for counting were not routinely taken at this step in an effort to obtain the maximum recovery for the third HPLC purification step. Recovery of the carrier peptides, from the initial $25 \mu \mathrm{g}$ through this step were: $\mathrm{AVP}=59 \%(n=8), \mathrm{OXY}=58 \%(n=15)$. Again, no significant difference was observed in the recovery of carrier from the three regions.

The final HPLC purification step used an ion-pairing buffer in the separation system in an effort to achieve an additional means of separation from any contaminants that might still be present. Figure 4 depicts the UV and radioactive profiles from the three regions for OXY-sulfoxide. There was a clear peak of radioactivity which co-migrated exactly with the oxidized carrier peptide from each of the three regions. Analogous results were obtained for AVP-sulfoxide. The radioactivity plotted corresponds to the total radioactivity recovered in each sample after this step, corrected only for quenching of the scintillation process. The recovery of the initially added carrier peptide through this step was: $\mathrm{AVP}=35 \%$ $(n=12)$ and $\mathrm{OXY}=36 \%(n=15)$.

Additional support for the purity of the radiolabeled peptides after the final HPLC purification step is given in Table I. These data demonstrate that for both the $\left[{ }^{35} \mathrm{~S}\right] \mathrm{AVP}$ and the $\left[{ }^{35} \mathrm{~S}\right] \mathrm{OXY}$ the radiolabeled peptides displayed constant specific activity between the second and third HPLC purification steps for both the PVN and

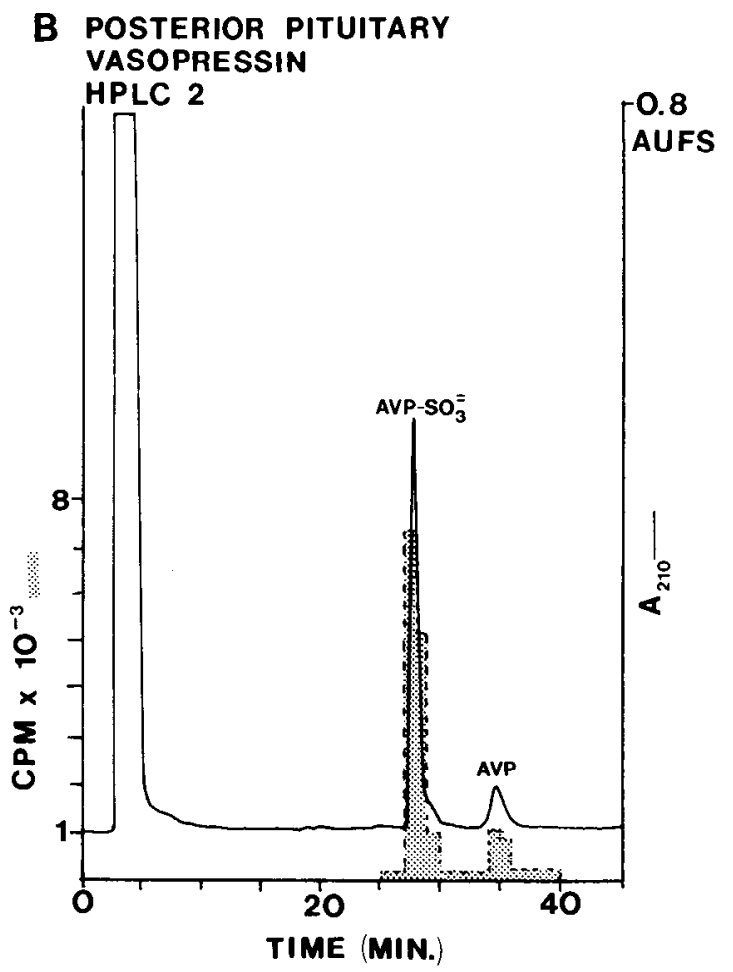

Figure 2. Chromatograms of the performic acid-oxidized AVP samples taken from the first HPLC. The gradient is an exponential gradient of Buffer $\mathrm{A} / \mathrm{CH}_{3} \mathrm{CN}$ from 5 to $18 \% \mathrm{CH}_{3} \mathrm{CN}$ over $40 \mathrm{~min}$. Axes are as in Figure 1 . $A$, PVN sample; $B$, posterior pituitary sample. In this and all subsequent purification steps, all of the sample from the previous purification step (less any aliquot removed for radioactivity determination) was lyophilized and then applied to the next HPLC system. 

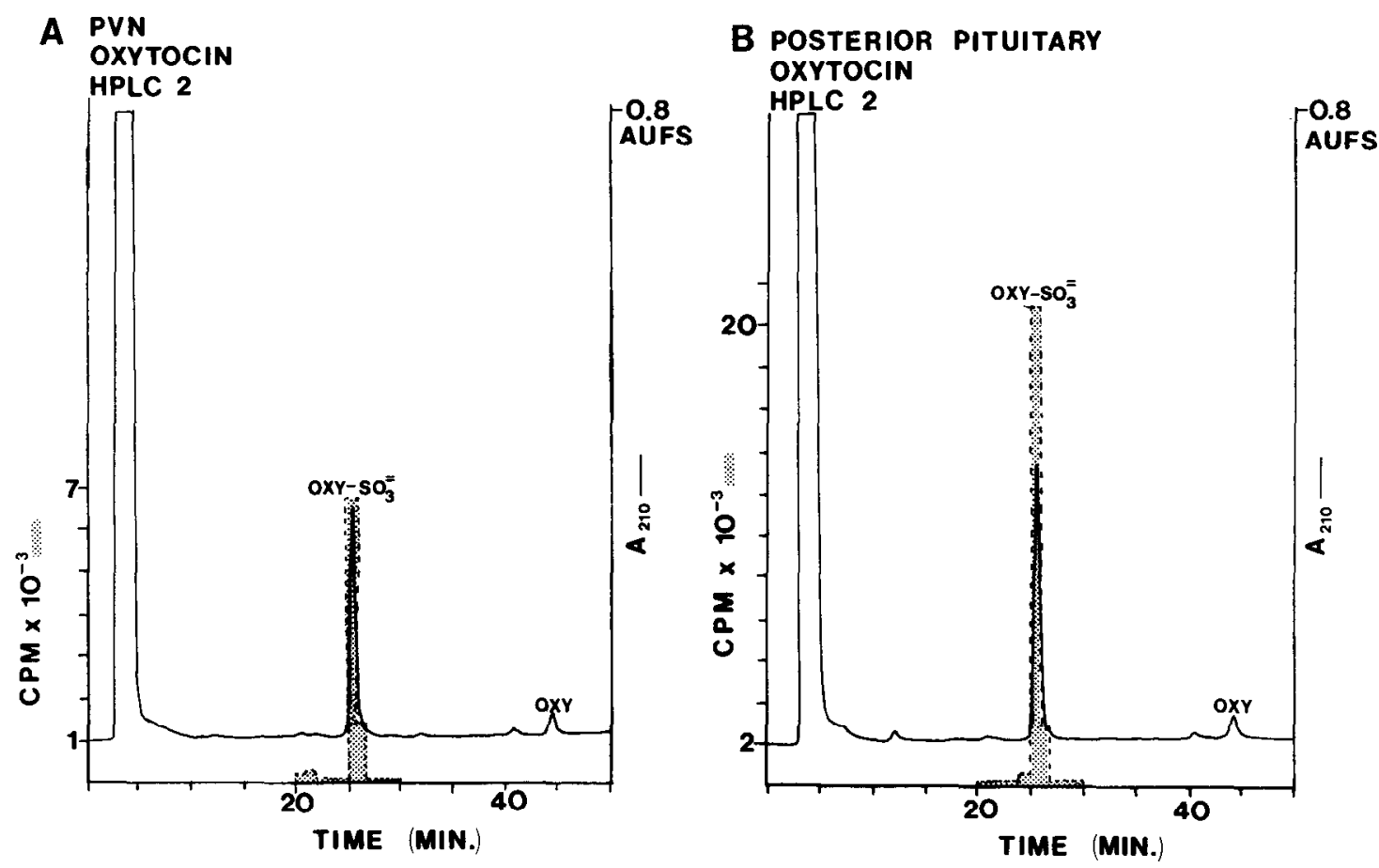

Figure 3. Chromatograms of the performic acid-oxidized OXY samples taken from the first HPLC. The gradient is an exponential gradient of Buffer $\mathrm{A} / \mathrm{CH}_{3} \mathrm{CN}$ from 5 to $22 \% \mathrm{CH}_{3} \mathrm{CN}$ over 40 min. Axes are as in Figure 1. A, PVN sample; $B$, posterior pituitary sample.
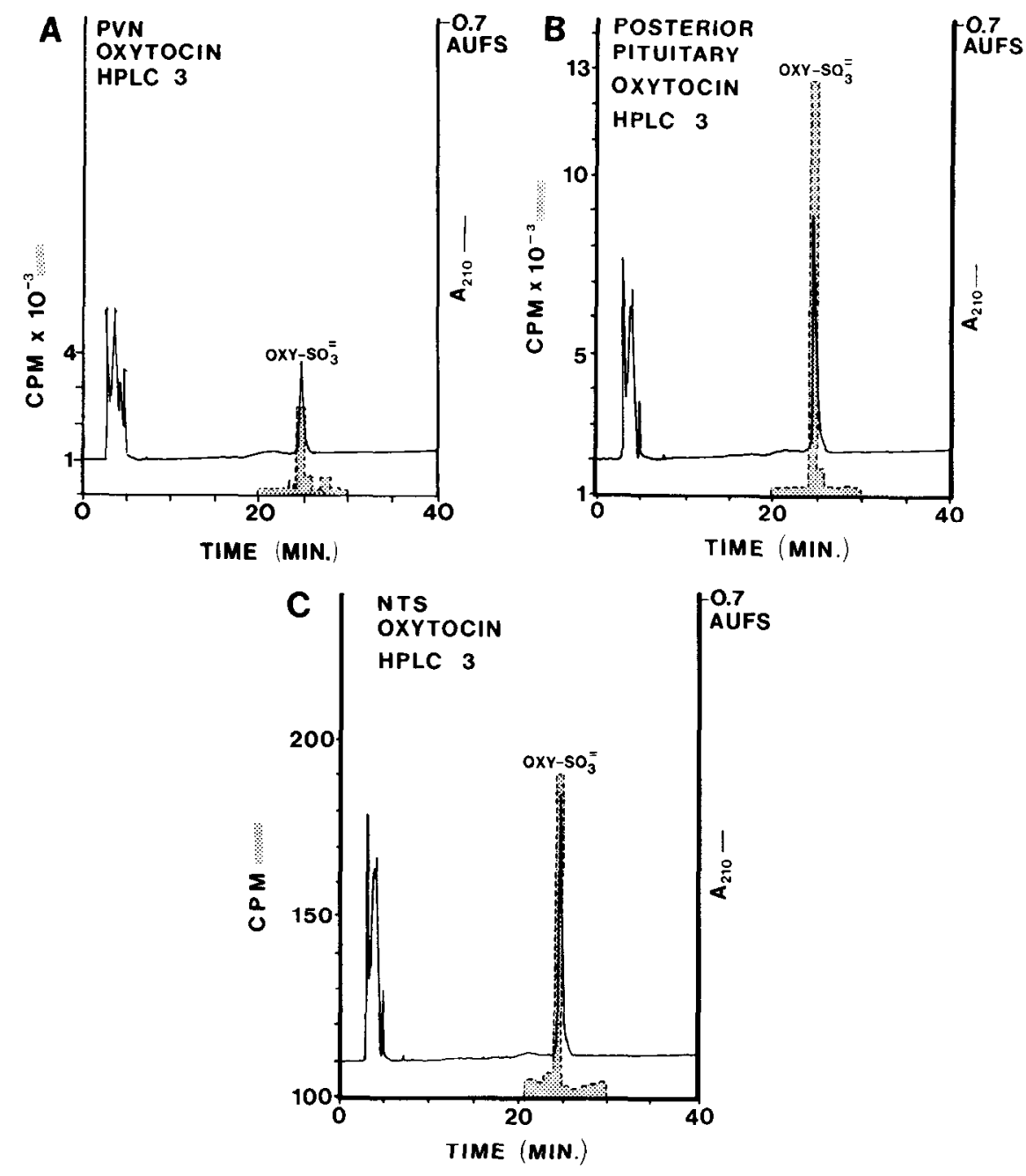

Figure 4. Chromatograms of the performic acid-oxidized OXY samples taken from the second HPLC purification (see Fig. 3). The gradient is a linear gradient of TEAP/ $\mathrm{CH}_{3} \mathrm{CN}$ from 5 to $30 \% \mathrm{CH}_{3} \mathrm{CN}$ over 40 min. Axes are as in Figure $1 . A$, PVN sample; $B$, posterior pituitary sample; $C$, NTS sample. In each case a peak of radioactivity co-eluted with the purified carrier peptide. 
the posterior pituitary samples. As mentioned above, we were unable to obtain specific activity data for the NTS samples at the second HPLC purification step. However, Table I demonstrates the severalfold purification of the radiolabeled peptides in the NTS samples between the first and the final HPLC separations.

Because the NTS lies at the dorsal surface of the brainstem, one potential means by which ${ }^{35}$ S-labeled peptides could be harvested from the NTS tissue samples is if the peptides were present in cerebrospinal fluid

TABLE I

Specific activities of $\left.P^{35} S\right] A V P$ and ${ }^{35} S J O X Y$

The values given are the ratio of the ${ }^{35} \mathrm{~S}$ counts per minute divided by the area of the carrier peptide peak. Each of the HPLC conditions is given under "Materials and Methods." For the NTS samples too little radioactivity was present in the samples to permit a determination of the radioactivity in the sample after both HPLC 2 and HPLC 3.

\begin{tabular}{cll}
\hline & \multicolumn{1}{c}{ AVP } & \multicolumn{1}{c}{ OXY } \\
\hline PVN & & \\
HPLC 1 & 0.00637 & 0.0106 \\
HPLC 2 & 0.00304 & 0.00754 \\
HPLC 3 & 0.00318 & 0.00722 \\
Posterior pituitary & & \\
HPLC 1 & 0.00691 & 0.0174 \\
HPLC 2 & 0.00634 & 0.0182 \\
HPLC 3 & 0.00667 & 0.0169 \\
NTS & & \\
HPLC 1 & 0.00046 & 0.00100 \\
HPLC 2 & ND $^{a}$ & ND \\
HPLC 3 & 0.00004 & 0.00022 \\
\hline
\end{tabular}

${ }^{a} \mathrm{ND}$, not determined.
(CSF) in the tissue. Although our calculations, based on available data for AVP and OXY concentrations in CSF (Perlow et al., 1982), suggested that at most $0.1 \mathrm{cpm}$ of labeled peptide would be present in CSF in the NTS tissue punch, a knife-cut lesion experiment was performed to demonstrate transport of labeled peptide from the PVN to the NTS.

Thus, a single sagittal knife cut was made at the level of the NTS between the nucleus and the solitary tract. The animals were allowed to recover for 10 to 14 days before cannulation and label administration. Figure 5 shows the radioactivity profile from the second HPLC purification step of AVP-sulfoxide for the left (control) and right (lesion) NTS tissue punch. The values plotted have been normalized for the slight differences in recovery of carrier peptide and for the radioactivity present in each PVN homogenate. As the figure illustrates, there was a significant depletion ( $75 \%$ ) of labeled peptide that could be purified from the NTS punch on the lesioned side. A similar depletion of OXY-sulfoxide was also observed. No depletion was found in the sham-operated animals.

The co-translation of vasopressin-neurophysin I and of oxytocin-neurophysin II is now well established for the neurohypophysial system. Therefore, our ability to detect biosynthetically labeled oxytocin and vasopressin in the NTS implies that each of the neurophysins could also be present in the brainstem, if processing in this projection is similar to that for the neural lobe projection. Table II shows the results of experiments in which radiolabeled peptide which co-migrated with the added carrier neurophysin was purified by two successive
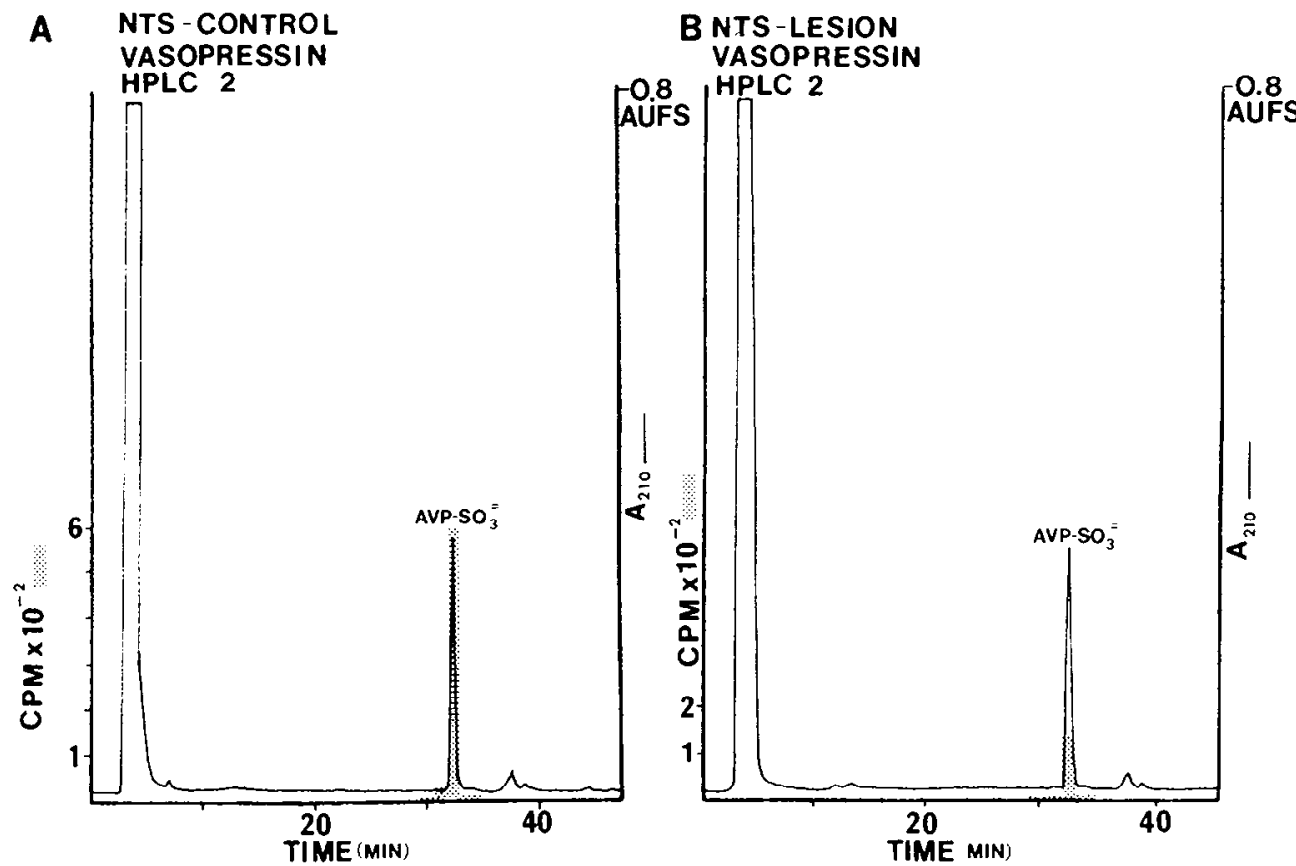

Figure 5. Chromatograms of the performic acid-oxidized AVP samples taken from the first HPLC purification and applied to the exponential gradient system described in Figure 2. The samples were purified from a rat with a unilateral lesion of the hypothalamic fibers leading to the NTS then cannulated and labeled as described under "Materials and Methods." Axes are as in Figure 1. The counts per minute plotted have been normalized to correct for the slight differences in recoveries of the carrier peptide between the two samples (Control $=69 \%$ recovery of the initially added carrier, and Lesion $=65 \%$ recovery) and the slight differences in the amount of radioactivity delivered through each cannula, as determined by total acid-soluble radioactivity. $A$, Control (unoperated) NTS sample; $B$, lesioned NTS sample. 
TABLE II

Specific activities of ${ }^{35} \mathrm{~S}$-neurophysins

The specific activities given are as defined in Table I. The conditions for the HPIC separation systems are given under "Materials and Methods."

\begin{tabular}{lccccc} 
& \multicolumn{2}{c}{ NP I } & & \multicolumn{2}{c}{ NP II/III } \\
\cline { 2 - 3 } \cline { 5 - 6 } & $\begin{array}{c}\text { Posterior } \\
\text { Pituitary }\end{array}$ & NTS & & $\begin{array}{c}\text { Posterior } \\
\text { Pituitary }\end{array}$ & NTS \\
\hline HPLC 1 & 0.05 & 0.0014 & & 0.11 & 0.00214 \\
HPLC 2 & 0.05 & 0.0016 & & 0.12 & 0.00277 \\
\hline
\end{tabular}

HPLC gradient systems. Although the recovery of the neurophysins was lower than that of the nonapeptides for the NTS samples, ${ }^{35} \mathrm{~S}$ radioactivity which co-migrated with carrier rat neurophysin proteins in two successive HPLC systems was observed for both the pituitary and NTS samples. As shown in Table II, the specific activity of the neurophysins was constant between the first and second HPLC systems.

Further verification of the presence of radiolabcled neurophysins in the NTS was provided by immunoprecipitation experiments in which the fractions corresponding to the elution positions of the neurophysins in the first HPLC separation were incubated with antineurophysin antiserum and the immunoprecipitated proteins were analyzed on the exponential gradient HPLC separation system. Again, radiolabeled proteins which co-migrated with carrier neurophysins were observed (Fig. 6).

${ }^{35}$ S-radioactivity, which co-purified with carrier neurophysins through the two HPLC systems described above, was then taken for trypsin treatment under nonreducing conditions. These conditions were chosen to permit all of the neurophysin-associated ${ }^{35} \mathrm{~S}$ radioactivity present in the NTS samples to be contained within a single tryptic peptide. These conditions were chosen because complete tryptic digestion would have resulted in four ${ }^{35} \mathrm{~S}$-tryptic peptides with a ratio of radioactivity of 5:4:3:2. Due to the low amount of $\left[{ }^{35} \mathrm{~S}\right]$ neurophysin present in the NTS samples, radioactivity measurements of these four ${ }^{35} \mathrm{~S}$ peptides would have been difficult. Thus, under the trypsin treatment conditions chosen, the NTS samples displayed a carrier peptide profile and a radioactivity profile which were identical to the profiles from the pituitary samples. The neurophysin I samples showed UV and ${ }^{35} \mathrm{~S}$ peaks at $52.1 \mathrm{~min}$ and $56.7 \mathrm{~min}$, consistent with the elution positions of digested and native neurophysin I, and the neurophysin II + III samples showed UV and ${ }^{35} \mathrm{~S}$ peaks at $47.8 \mathrm{~min}$ and $49.8 \mathrm{~min}$, again consistent with the elution positions of digested and native neurophysin II + III.

\section{Discussion}

The data presented above demonstrate the feasibility of studying the biosynthesis and transport of the neurohypophysial hormones AVP and OXY to projection sites from the PVN to autonomic regulatory and other sites within the central nervous system (CNS). Using the stereotaxic cannulation and osmotic minipump delivery system and the pulse-labeling paradigm, we have shown that radioactivity labeled AVP, OXY, and their neurophysins are synthesized in the PVN and delivered to the NTS in the medulla. The identification of each of these peptide species is based on rigorous, sequential biochemical criteria and does not rely on immunological or affinity-binding techniques. The HPLC separation systems used here employ both linear and exponential gradient acetonitrile elution coupled with chemical derivatization of the peptides. In addition, both standard (phosphate) and ion-pairing (TEAP) buffer systems were used, which allow for elution of the peptides from the reverse-phase columns based on different hydrophobic and ionic properties. Furthermore, the identification of the neurophysins is based not only on acid solubility, cysteine content, and HPLC elution characteristics but also on tryptic mapping studies. These purification techniques also are both rapid and achieve separation of the peptides of interest from contaminants with excellent recovery at each step.

Several recent anatomical studies (e.g., Swanson and Kuypers, 1980; Sawchencko and Swanson, 1982a) have demonstrated the presence of AVP-, OXY-, and neurophysin-like immunoreactivity in the NTS; however, the data above are the first biochemical demonstration to support the conclusion that these immunologically identified peptides are chemically similar, if not identical, to the neurohypophysial peptides released from the posterior pituitary. The absolute amount of radioactivity in each of the peptides harvested from the NTS was significantly less than that harvested from the pituitary $(<1 \%)$. However, it is highly unlikely that this radioactivity could be accounted for by uptake of the peptides

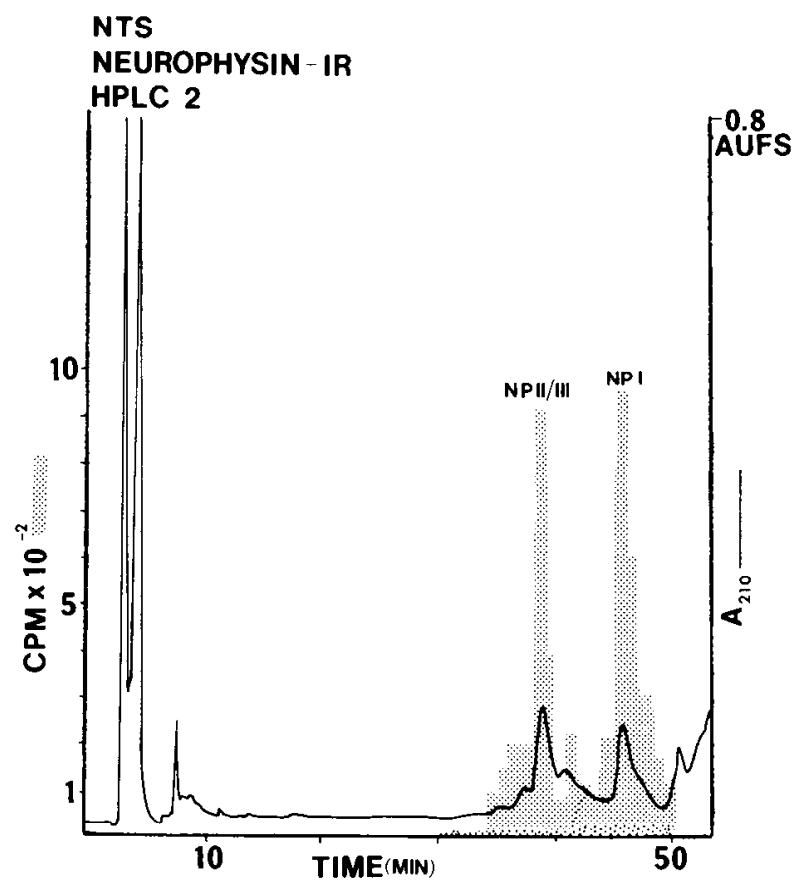

Figure 6. Chromatogram of the proteins eluted from the Protein A-Sepharose column after immunoprecipitation of the fractions from the first HPLC purification step of the NTS tissue homogenates, corresponding to the elution position of the neurophysins, with anti-neurophysin antibody. The HPLC system used is the exponential gradient shape described under "Materials and Methods" for the separation of the tryptic fragments of neurophysin. The elution positions of the added carrier neurophysins II + III $(N P I I / I I I)$ and I $(N P I)$ are marked. 
from the CSF, since a unilateral lesion of the solitary tract resulted in depletion of the hormones harvested from the NTS ipsilateral to the lesion but not contralateral to the lesion. Thus, the ability to detect the peptides in the NTS was dependent upon the localized administration of high specific activity ${ }^{35}$ S $]$ cysteine and the use of relatively large amounts of radioactivity $(0.5 \mathrm{mCi} /$ animal). The relative magnitude of the radioactivity recovered from the NTS versus the pituitary samples could be a reflection of a number of variables, such as the placement of the cannulae or the relative number of parvocellular neurons projecting to the descending sites versus the number of magnocellular neurons projecting to the neural lobe. Another possibility is that the radioactivity profiles reflect the different requirements of the neuronal projections and the endocrine projection. In these initial experiments defining OXY, AVP, and neurophysin biosynthesis and transport, the 12-hr pulselabeling time was chosen as a compromise for the unknown times of synthesis and processing of the neuropeptide precursors, the transport of the peptides over the 15-mm distance from the PVN to the NTS, and the accumulation of radiolabeled peptides in the terminal field. Preliminary studies suggest that the neuropeptides, in fact, are transported from the PVN to the NTS via the fast component of axonal transport (J. D. White, J. E. Krause, and J. F. McKelvy, manuscript in preparation). Thus, further, detailed studies examining the possible physiological regulation of the biosynthesis of these neuropeptides will require the determination of the pulse-chase parameters that yield the maximum amount of radiolabeled peptide in the NTS.

The avidity with which the neurophysin proteins bound to the HPLC column resulted in virtually complete purification of these proteins in the first HPLC system. This is attested to by the observed ratios of nonapeptide to neurophysin radioactivity seen in the pituitary samples (Fig. 1) and by the constant specific activity between the two successive HPLC steps exhibited by the neurophysin proteins for all samples (Table II). Additionally, the anti-neurophysin immunoprecipitation/HPLC analysis and the trypsin digestion studies also provide strong evidence for the transport of neurophysin proteins, as well as the nonapeptides, to the brainstem. Using the HPLC systems described above, we are unable to separate neurophysin II from neurophysin III, although each is specifically associated with OXY (Sunde and Sokol, 1975; Pickering and North, 1982). Studies now in progress are designed to determine the possible metabolic relationship between these two neurophysins in the pituitary and brainstem projection systems.

The above data have also demonstrated that AVP, $\mathrm{OXY}$, and neurophysin synthesis in the PVN and transport to the pituitary can be investigated in a single animal. These data differ from previous studies of hypothalamic synthesis and transport of the nonapeptides (Sachs et al., 1969; Brownstein et al., 1980; FrancoBourland and Fernstrom, 1981) in several respects. In the present study biosynthesis was studied in PVN neurons, not the SON or the entire hypothalamus, in individual animals in a normal state of hydration and relied solely on sequential chemical criteria for the identifica- tion of the hormones and the neurophysins. These data also demonstrate that studies on the physiological regulation of AVP and OXY biosynthesis for both endocrine and autonomic projections are now possible using individual animals. One limitation of the present technique of in vivo radiolabeling to examine the possible physiological regulation of neuropeptide biosynthesis is that any measurement of peptides in a given synaptic field will represent the sum of peptide synthesis and peptide release and turnover. However, despite this limitation, this technique can, for example, estimate the minimum change in the rate of synthesis of a given neuropeptide for a physiologically activated system when an appropriately short time for synthesis and transport is chosen. Such rate measurements are not possible using static measurements, such as determining peptide levels by radioimmunoassay. Furthermore, at any time, the examination of the radiolabeled peptides present within a given synaptic field may yield information regarding differential post-translational processing of neuropeptide precursors by a specific nucleus.

The PVN has long been known to be responsive to changes in osmotic balance and blood pressure and to release AVP, OXY, and neurophysin from nerve terminals in the posterior pituitary into the general circulation to maintain the osmotic balance of the animal (see Bie, 1980, for a review). The demonstration of AVP, OXY, and neurophysins in PVN neurons that project to the NTS suggest that these peptides may play a role within the CNS in the regulation of blood pressure. The NTS has been shown to be a rich area of baroreceptor input in a number of mammalian species (e.g., see Hilton and Spyer, 1980; Czachurski et al., 1982, for a review). Berecek et al. (1982) have shown that electrical stimulation of the PVN produces a number of cardiovascular effects and that, in homozygous diabetes insipidus rats (genetically unable to produce vasopressin), there was little or no cardiovascular response to PVN stimulation. In further support of the hypothesis of CNS-mediated effects of AVP (and OXY) on blood pressure, it has been shown that intracerebroventricular administration of AVP caused short-latency increases in blood pressure in rats (Pittman et al., 1982). A more direct demonstration of the role of AVP and OXY (and neurophysins?) on blood pressure regulation in the NTS awaits detailed electrophysiological experiments as well as the demonstration of high-affinity receptors for AVP and OXY in the NTS. Additionally, monosynaptic noradrenergic connections have been demonstrated from the NTS to the PVN (Sawchencko and Swanson, 1982b). Catacholamines have been shown to affect the firing patterns of both AVP and OXY neurons in the PVN (see Poulain and Wakerly, 1982, for a review). Thus, the pathways have been demonstrated by which the PVN could receive input from and have influence upon neurons in the NTS which are responsible for blood pressure regulation.

The physiological role(s) of the neurophysins remains a mystery. The ability to harvest radiolabeled neurophysins from the NTS supports the hypothesis for a single prohormone, for each of the nonapeptides, which contains both the hormone and neurophysin. This is also consistent with the recent cDNA data of Land et al. $(1982,1983)$ which have shown a mRNA for the AVP- 
neurophysin precursor and for the OXY-neurophysin precursor in the calf and with the gene sequence data for the rat, which demonstrated that the vasopressin sequence was adjacent to the neurophysin sequence (Schmale et al., 1983). However, any physiological role for the neurophysins either in peripheral tissue or in the CNS remains to be determined. The demonstration of their transport to $\mathrm{OXY}$ and vasopressin terminal fields in the brainstem suggests a need to determine their effects on the excitability of postsynaptic neurons at this site.

\section{References}

Berecek, K. H., R. L. Webb, K. W. Barron, and M. J. Brody (1982) Vasopressin projections and central control of cardiovascular function. Ann. N. Y. Acad. Sci. 394: 729-734.

Bie, P. (1980) Osmoreceptors, vasopressin and control of renal water excretion. Physiol. Rev. 60: 961-1048.

Brownstein, M. J., J. T. Russell, and H. Gainer (1980) Synthesis, transport and release of posterior pituitary hormones. Science 207: 373-378.

Czachurski, J., K. J. Lackner, D. Ockert, and H. Seller (1982) Localization of neurons with baroreceptor input in the medial solitary nucleus by means of intracellular application of horseradish peroxidase in the cat. Neurosci. Lett. 28: 133137.

Elliott, K. A. C. (1969) The use of brain slices. In Handbook of Neurochemistry, A. Lajtha, ed., Vol. 2, pp. 103-114, Plenum Press, New York.

Franco-Bourland, R. E., and J. D. Fernstrom (1981) In vivo biosynthesis of L- $\left.{ }^{33} \mathrm{~S}\right]$ cys-arginine vasopressin, -oxytocin, and -somatostatin: Rapid estimation using reversed phase high pressure liquid chromatography. Endocrinology 109: 1097-1106.

Hilton, S. M., and K. M. Spyer (1980) Central nervous regulation of vascular resistance. Annu. Rev. Physiol. 42: 399-411.

Hirs, C. H. W. (1967) Performic acid oxidation. Methods Enzymol. 11: 197-199.

Land, H., G. Schutz, H. Schmale, and D. Richter (1982) Nucleotide sequence of cloned cDNA encoding bovine arginine vasopressin-neurophysin II precursor. Nature 295: 299-303.

Land, H., M. Grez, S. Ruppert, H. Schmale, M. Rehbein, D. Richter, and G. Schutz (1983) Deduced amino acid sequence from the bovine oxytocin-neurophysin I precursor cDNA, Nature 302: 342-344.

McKelvy, J. F., J. E. Krause, and J. D. White (1983) Methods for the study of the biosynthesis of neuroendocrine peptides in vivo and in vitro. Methods Enzymol. 103: 511-523.

Perlow, M. J., S. M. Reppert, H. A. Artman, D. A. Fisher, S. M. Seif, and A. G. Robinson (1982) Oxytocin, vasopressin and estrogen-stimulated neurophysin: Daily patterns of concentration in cerebrospinal fluid. Science 216: 1416-1418.

Pickering, B. T., and W. M. North (1982) Biochemical and functional aspects of magnocellular neurons and hypothalamic diabetes insipidus. Ann. N. Y. Acad. Sci. 394: 72-81.

Pittman, Q. J., D. Lawrence, and L. McLean (1982) Central effects of arginine vasopressin on blood pressure in rats. Endocrinology 110: 1058-1060.

Poulain, D. A., and J. B. Wakerley (1982) Electrophysiology of hypothalamic magnocellular neurones secreting oxytocin and vasopressin. Neuroscience 7: 773-808.

Sachs, H. (1969) Neurosecretion. In Handbook of Neurochemistry, A. Lajtha, ed., Vol. 4, pp. 323-428, Plenum Press, New York.

Sachs, H., C. P. Fawcett, Y. Takabatake, and R. Portanova (1969) Biosynthesis and release of vasopressin and neurophysin. Recent Prog. Horm. Res. 25: 447-491.

Sawchencko, P. E., and L. W. Swanson (1982a) Immunohistochemical identification of neurons in the paraventricular nucleus of the hypothalamus that project to the medulla or to the spinal cord in the rat. J. Comp. Neurol. 205: 260-272.

Sawchencko, P. E., and L. W. Swanson (1982b) Organization of noradrenergic pathways from the brainstem to the paraventricular nucleus and the supraoptic nucleus. Brain Res. Rev. 4: 275-325.

Scharrer, E., and B. Scharrer (1954) Hormones produced by neurosecretory cells. Recent Prog. Horm. Res. 10: 183-240.

Schmale, H., S. Heinsohn, and D. Richter (1983) Structural organization of the rat gene for the arginine vasopressinneurophysin precursor. EMBO J. 2: 763-767.

Sinding, C., A. G. Robinson, and S. M. Seif (1980) Levels of neurohypophysial peptides in the rat during the first month of life. II. Response to physiological stimuli. Endocrinology 107: $755-760$.

Sunde, D. A., and H. W. Sokol (1975) Quantification of rat neurophysins by polyacrylamide gel electrophoresis (PAGE): Application to the rat with hereditary hypothalamic diabetes insipidus. Ann. N. Y. Acad. Sci. 248: 345-364.

Swanson, L. W., and H. G. J. M. Kuypers (1980) The Paraventricular nucleus of the hypothalamus: Cytoarchitectonic subdivisions and organization of projections to the pituitary, dorsal vagal complex and spinal cord as demonstrated by retrograde fluorescence double-labelling methods. J. Comp. Neurol. 194: 555-570. 